\title{
Grain boundaries with octagonal defects in graphene nanoribbons and nanotubes
}

\author{
M. Pelc, ${ }^{1}$ L. Chico, ${ }^{2}$ A. Ayuela, ${ }^{3}$ and W. Jaskólski ${ }^{1}$ \\ ${ }^{1}$ Institute of Physics, Faculty of Physics, Astronomy and Informatics, Nicolaus Copernicus University, Grudziadzka 5, 87-100 Toruń, Poland \\ ${ }^{2}$ Instituto de Ciencia de Materiales de Madrid (ICMM), Consejo Superior de Investigaciones Científicas (CSIC), \\ C/Sor Juana Inés de la Cruz 3, 28049 Madrid, Spain \\ ${ }^{3}$ Centro de Física de Materiales, CFM-MPC CSIC-UPVIEHU, Donostia International Physics Center (DIPC), \\ Departamento de Física de Materiales, Facultad de Químicas, UPV-EHU, 20018 San Sebastián, Spain
}

(Received 25 January 2013; published 15 April 2013)

\begin{abstract}
We study graphene nanoribbons and carbon nanotubes containing ordered defect lines built of octagonal rings. We show that octagonal defect lines are a robust source of state localization at the Fermi energy, in some cases leading to spontaneous magnetization. We also prove that the localization at chains of octagons is a consequence of the zigzag nature of the graphene edges forming the defect lines.
\end{abstract}

DOI: 10.1103/PhysRevB.87.165427

PACS number(s): 73.22.-f, 73.63.-b

\section{INTRODUCTION}

The experimental observation of grain boundaries in graphene, ${ }^{1-3}$ either disordered ${ }^{1}$ or as periodic extended defect lines, ${ }^{2}$ constitutes a great motivation for the theoretical study of such defect arrangements. Recently, much effort has been devoted to investigating the structural properties and atomic formation of such extended defects. ${ }^{4-6}$ In fact, grain boundaries are especially interesting because they can strongly modify the electronic properties of graphene and carbon-based structures. This is because they present localized states at the Fermi level, which influence their transport and magnetic properties..$^{711}$

Grain boundaries in graphene can be seen as defect lines formed at junctions between sheets of graphene or graphene ribbons. Experimental techniques nowadays allow the patterning of graphene into nanometer-size ribbons with well-controlled size and shape of the edges. ${ }^{12,13}$ Graphene nanoribbons (GNRs) with smooth edges can also be obtained by unzipping carbon nanotubes (CNTs) ${ }^{14,15}$ So it can be envisioned that by manipulating graphene nanoribbons and their edges, they could be connected, forming lines of topological defects at their interfaces. For example, an armchair edge can be joined to a zigzag one by a line of pentagon-heptagon (5-7) defects. ${ }^{16}$ Interface states that localize at such junctions have been customarily related to these topological defects. However, it has been recently shown that such states do not originate from the 5-7 defects, but are due to the edge-localized states, which in turn stem from the zigzag edges of the joined systems. ${ }^{16,17}$

In graphene nanoribbons, and generally in any graphene structure with edges, the condition for the existence of edge-localized states at the Fermi energy, i.e., the vanishing of the sum of the wave function coefficients at all nearestneighbor nodes around a given node, enforces localization of the corresponding wave function at only one sublattice. ${ }^{18}$ The presence of odd-numbered topological defects, which is typical at interfaces between different graphene structures, mixes the two graphene sublattices. As a consequence, the localized states move away from the Fermi energy. ${ }^{16}$ This is the case of 5-7 defects. However, interfaces between two zigzag ribbons can be made with octagonal rings accompanied by pentagons. Such interfaces are just graphene defect lines built of octagons and pentagons. In these systems the interface localized states have energies at the Fermi level, so they can strongly influence the electronic and magnetic properties. For example, it has been recently shown that these double pentagon-octagon junctions may act as quasi-one-dimensional metallic wires. ${ }^{2}$ Electronic and magnetic properties of ribbons with octagon-pentagon line defects are now being intensively investigated. ${ }^{19-22}$

In this work we study the appearance of localized states in graphene-based systems with octagonal defects. We investigate octagonal defect lines appearing both in graphene ribbons and in carbon nanotubes, which can be considered as rolled-up ribbons. To this end, we bring into play previous findings on localized states on graphene edges. ${ }^{17}$ We show that zero-energy states localized at octagonal defect lines have their origin in the edge-localized states of ribbons. In some instances, these defect lines may give rise to spontaneous magnetization. We employ a $\pi$-electron tight-binding (TB) approximation, including electron interaction effects by means of the Hubbard model. A Green function matching approach is used to obtain the local density of states (LDOS). ${ }^{23}$ Our main results are the following:

(i) Octagonal defects localize states at the Fermi energy even when the two graphene sublattices are mixed. In some cases, they may induce spontaneous magnetization. This is at variance with defects composed of pentagons and heptagons.

(ii) We fully explain this localization using the hybridization rules introduced for GNRs with arbitrary edges and presented in Ref. 17.

(iii) Junctions between zigzag $(n, 0)$ nanotubes ${ }^{24}$ made of octagons and pentagon pairs also present interface localized states. The number of such states and their energies depend on $n$, i.e., on the diameter of the nanotube. Their origin and size dependence are explained by the application of a folding rule (Ref. 16) to a graphene sheet with an octagonal defect line.

\section{MODEL AND METHOD}

All the calculations are performed within the $\pi$-electron tight-binding approximation. The electron interaction effects are taken into account within the Hubbard model. The tightbinding approach has been shown to be adequate for the 
modeling of the electronic properties around the Fermi energy in carbon nanotube superlattices, where localized states may also appear. ${ }^{25,26}$ In order to see the role of electron-electron interactions in the zero-energy states, we compare the oneelectron tight-binding results with those including a Hubbard term.

The Hubbard Hamiltonian in a mean-field approximation is given $b^{27}$

$$
H=t \sum_{\langle i, j\rangle, \sigma} c_{i \sigma}^{\dagger} c_{j \sigma}+\text { H.c. }+U \sum_{i}\left(n_{i \uparrow}\left\langle n_{i \downarrow}\right\rangle+\left\langle n_{i \uparrow}\right\rangle n_{i \downarrow}\right),
$$

where $c_{i \sigma}^{\dagger}\left(c_{i \sigma}\right)$ are the creation (annihilation) operators for electrons with spin $\sigma$ at site $i ;\langle i, j\rangle$ indicates that the sum is restricted to nearest neighbors; the index $i$ runs over all the nodes in the unit cell; and the arrows correspond to the two possible spin states. The first term is the single-particle tight-binding Hamiltonian, while the second one represents the on-site Coulomb repulsion. The hopping parameter $t$ is set equal to $-2.7 \mathrm{eV}$ and the on-site Coulomb repulsion parameter $U$ is taken as $3 \mathrm{eV}$. Since the expectation values of the spinresolved densities at site $i,\left\langle n_{i, \sigma}\right\rangle=\left\langle c_{i, \sigma}^{\dagger} c_{i, \sigma}\right\rangle$, depend on the eigensolutions of the Hamiltonian, the above equation is solved iteratively by means of the self-consistent field method.

We use a single and constant value of the hopping parameter $t=-2.7 \mathrm{eV}$, although the carbon-carbon bond length changes near the defects. ${ }^{28}$ We have checked that the usage of bondlength-dependent $t$ changes negligibly the calculated energy spectra and has almost no effect on flatbands close to the Fermi level, in agreement with previous calculations. ${ }^{26}$

A comment is required as to the on-site Coulomb repulsion parameter $U$. It is customary to choose a value that fits the gap of zigzag ribbons in density functional theory (DFT) calculations. By fitting to local density approximation (LDA) results, the value $U=2 \mathrm{eV}$ was suggested in Ref. 29. However, calculations performed beyond DFT in Ref. 30 using the GW approximation show that gaps are normally larger than those given by the LDA. We thus choose a higher $U=3 \mathrm{eV}$. Of course, we have checked that this choice does not change our results qualitatively with respect to the band structure and the magnetization. It confirms previous findings ${ }^{29,31}$ that the magnetization effects and general character of the bands are robust with respect to the value of $U$.

\section{GRAPHENE RIBBONS AND CARBON NANOTUBES WITH OCTAGONAL DEFECT LINES}

We begin by studying a zigzag GNR (ZGNR) with a defect line composed only of octagons, as shown in Fig. 1(a). Pentagons will be introduced at a later stage by connecting every second octagon, as depicted in Fig. 1(b). Additionally, we will also consider rolled-up systems, i.e., carbon nanotubes, which allow us to get rid of the zigzag-edge-localized states. We will analyze the differences among these closely related structures in order to elucidate the role of topological defects in their electronic properties. Finally, we will show that the appearance of zero-energy states localized at octagonal defects can be explained without performing calculations, but taking into account only the topology of the edges forming the octagonal defect lines. We will do it by applying the
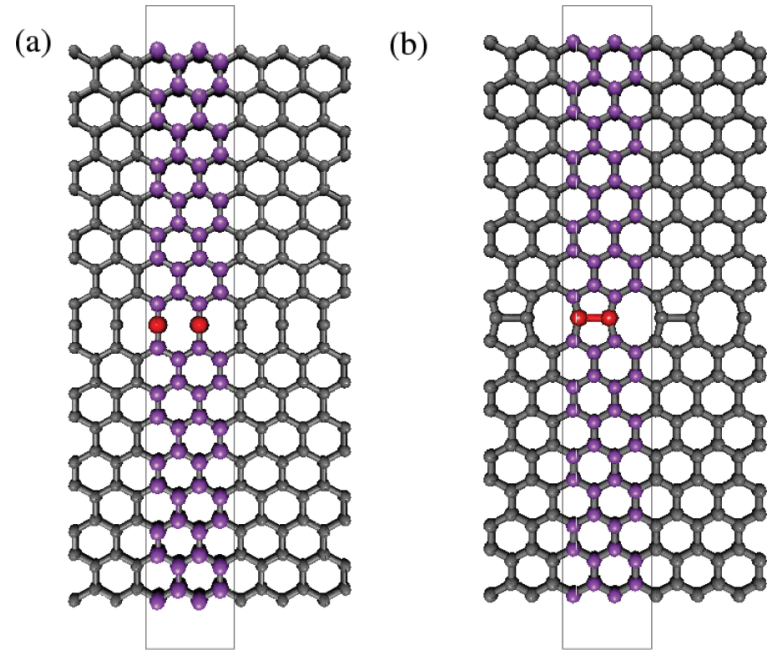

FIG. 1. (Color online) ZGNRs with defect lines composed of (a) consecutive octagons and (b) octagons with an adjacent pair of pentagons. The ZGNRs extend horizontally. Rectangles mark the unit cells of ribbons including the defects. Klein nodes (see the text) in the unit cells are highlighted in red.

rules introduced in Ref. 17 to count the edge-localized states at arbitrary GNRs. Notice that the model employed in the above-mentioned work (a one-orbital TB Hamiltonian) does not take into account spin degeneracy, so when comparing with the Hubbard calculation we should double the degeneracy of all the TB bands.

\section{A. Defect lines made of adjacent octagons}

First we consider a ZGNR with a defect line made of octagons only, as shown in Fig. 1(a). The unit cell of investigated ribbon [marked with a rectangle in Fig. 1(a)] contains 58 atoms. It has been chosen to be the same as in Ref. 2.

Such ribbon may be obtained by connecting two 8ZGNRs, $^{32}$ one of which has a Klein-like edge ${ }^{17}$ at one side, as shown in Fig. 1(a). Henceforth we will refer to these atoms as Klein nodes, even after their connection to form the octagons. This structure is less stable than the one experimentally observed, ${ }^{2}$ in which every second octagon is reconstructd into a pair of pentagons [see Fig. 1(b)]. However, this is an ideal model helpful in understanding localization effects at the octagonal defects. Notice that this system can be divided into two bipartite lattices. Consequently, the electronic spectrum within a $\pi$-orbital nearest-neighbor TB model has electron-hole symmetry, as can be seen in the energy bands shown in Fig. 2(a). Although there is no sublattice mixing, the defect line makes both zigzag edges belong to the same sublattice, differently from a defect-free zigzag ribbon. This introduces an imbalance between atoms belonging to opposite sublattices, with an excess of two atoms per unit cell in the sublattice to which the edges belong. Therefore, according to Lieb's theorem, ${ }^{33}$ there should be a net magnetic moment of two Bohr magnetons in this system, as we will corroborate below.

The wave functions corresponding to the four zero-energy bands at $k=0$, calculated in the TB model without spin, are 
(a)

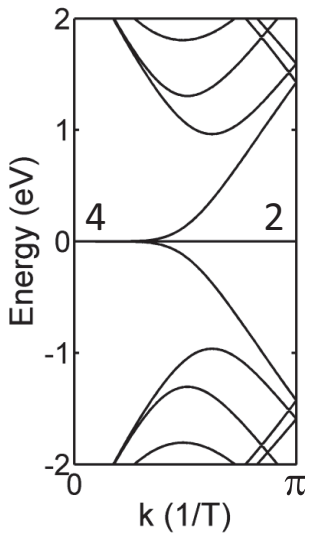

(b)

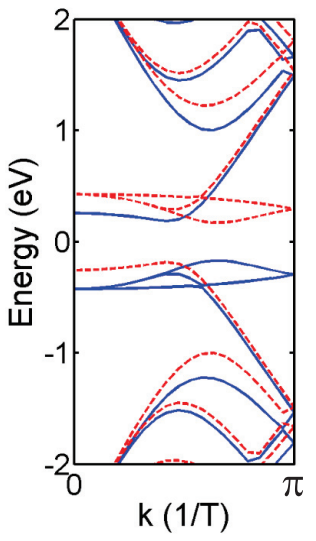

(c)

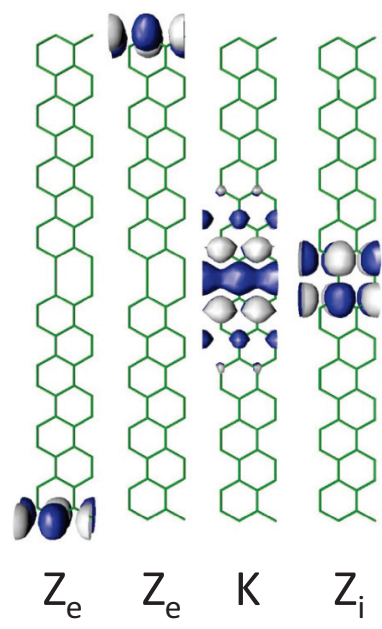

FIG. 2. (Color online) (a) Energy bands of a ZGNR with a defect line composed of octagons, corresponding to two 8-ZGNRs joined by an extra row of Klein-like atoms, as depicted in Fig. 1(a), calculated within the TB approximation without considering spin. The wave vector $k$ is given in units of $1 / T$, where $T$ is the length of the unit cell. The degeneracies of $E=0$ bands at $k=0$ and $k=\pi$ without considering spin are indicated in the figure. (b) Energy spectrum for the same ribbon calculated including electron-electron interactions by means of a Hubbard model. Blue solid lines denote spin-up bands; red dashed lines show the spin-down bands. The Fermi energy is at $0 \mathrm{eV}$ in both cases. (c) TB wave functions corresponding to the four zeroenergy bands at $k=0$. Blue (dark gray) and white colors represent positive and negative signs of the wave function, respectively.

presented in Fig. 2(c). Those labeled by $Z_{e}$ belong to two states localized at the outer (external) zigzag edges of the ribbon. The other two flatbands, which extend over the entire Brillouin zone (BZ), have their wave functions localized at the octagonal defects, but in opposite sublattices. One of them, labeled $K$, looks like a state localized mainly at the Klein nodes. ${ }^{17}$ Note that these Klein atoms have only two nearest neighbors, i.e., coordination number 2 . The other band is similar to the edge states localized in zigzag nanoribbons, but separated by the row of Klein atoms where the wave function has zero weight. It stems from the two zigzag edge states which are now in the inner part of the system, and thus labeled $Z_{i}$. Since the row of Klein atoms is in fact shared by both inner zigzag "edges," the electrons are also shared by them, being like a resonant state composed of a mixture of a double and a single bond in an aromatic ring. This idea is supported by the fact that the maximum of the wave function at these four inner zigzag nodes is half of the maximum value of the other three wave functions, so it can be considered that this resonant state is shared between the two inner zigzag edges.

Due to the sublattice imbalance, we expect a net magnetization in this system. Therefore, we take into account electron-electron interaction effects by employing the Hubbard model, in which the on-site Coulomb repulsion is included as outlined in Sec. II. The resulting bands are presented in Fig. 2(b). Blue solid lines correspond to spin-up (majority) bands and red dashed lines represent spin-down (minority) solutions. Since we include now the spin, the number of bands is doubled, as is clearly seen in the figure. At $k$ close to zero and for energies below the Fermi level, there are three nearly degenerate spin-up bands and one spin-down band. All the corresponding spin-up wave functions are localized at the same sublattice: one at the bottom zigzag edge, the second at the upper zigzag edge, and the third one at the internal Klein nodes of the octagonal defect. They closely resemble the first three TB functions shown in Fig. 2(c). The wave function of the spin-down band is localized at the inner zigzaglike nodes, i.e., on the opposite sublattice to that to which the Klein atoms belong. At $k>\frac{2 \pi}{3}$, there are two spin-up bands below $E_{F}$, while their spin-down counterparts are unoccupied. Therefore, the calculated spin polarization per unit cell is close to $2 \mu_{B}$ and the system presents spontaneous magnetization, consistent with the number given by Lieb's theorem. The validity of Lieb's theorem is quite remarkable because in this case it is applicable "without even the necessity of periodic lattice structure." 33

Now, let us roll up the system to get an armchair carbon nanotube with the line of octagonal defects along the tube axis. This can be viewed as an $(8,8)$ tube with an extra row of atoms along its length. In this way we get rid of the zigzag outer edges and their corresponding localized states. Since we have to connect the ZGNR outer edge nodes which belong to the same sublattice, the resulting nanotube does not have globally defined (i.e., valid throughout the entire tube) atom sublattices. Figure 3 shows the corresponding band structures, calculated within the TB approximation and including a Hubbard term, as well as the wave functions for the zero-energy states at $k=0$. Notice that, due to sublattice mixing, these bands do not have electron-hole symmetry. Since this system does not have zigzag edges, the two states that were previously localized at the outer edges in the associated ribbon, disappear in this case. The two surviving zero-energy bands are related to the line of octagonal defects. In fact, the wave functions at $k=0$ look exactly the same as the corresponding defect-related wave functions in the unrolled system, as can be seen in Fig. 3(c). This indicates that close to the octagons the two graphene sublattices are locally well defined, i.e., not mixed.

Moreover, there is a small energy gap between the flatband at the Fermi energy and the conduction bands, which is unusual for armchair tubes. However, since the flatband at $E=0$ is unoccupied, the nanotube preserves its metallic character. Nevertheless, in this case the electrons around the Fermi energy occupy the states around the Klein nodes. It is important to emphasize that the robust metallicity of armchair nanotubes is strongly disturbed because the line of defects perturbs the 


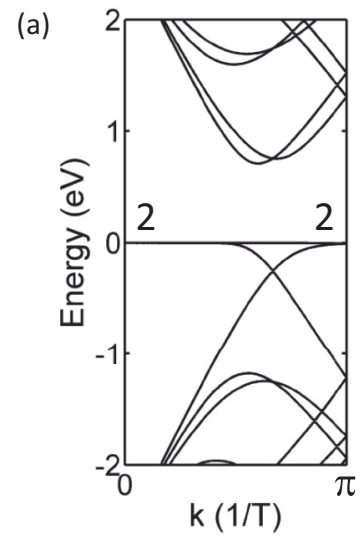

(b)

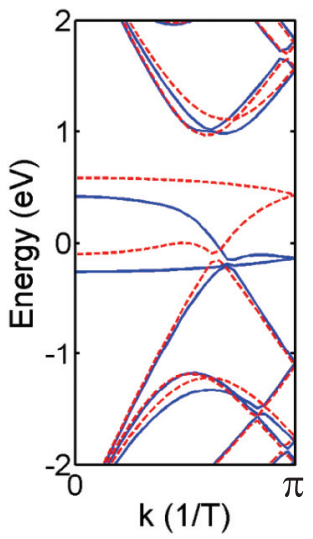

(c)

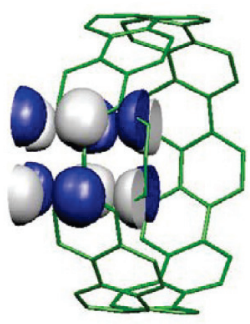

$\mathrm{Z}_{\mathrm{i}}$

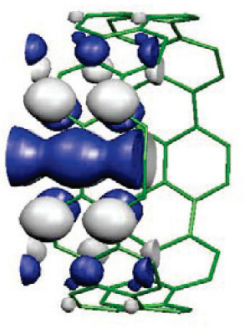

$\mathrm{K}$

FIG. 3. (Color online) (a) Energy bands of the armchair $(8,8)$ carbon nanotube with a defect line composed of octagons obtained by rolling up a ZGNR with octagonal defects, calculated with a TB model. Degeneracies are indicated without considering spin. (b) Energy spectrum for the same tube calculated with a Hubbard model. Blue solid lines, spin-up bands; red dashed lines, spin-down bands. (c) TB wave functions corresponding to two zero-energy bands at $k=0$. Blue (dark gray) and white colors represent positive and negative signs of the wave function, respectively.

nanotube wave functions, which are delocalized around the circumference in the case of the perfect nanotube.

The band structure calculated within the Hubbard model is presented in Fig. 3(b). Due to the sublattice mixing produced by rolling up the ribbon, Lieb's theorem does not apply in this case, so the magnetic moment has to be obtained from the electronic spectrum. For $k<\frac{2 \pi}{3}$ the two flatbands just below $E_{F}$ have different spin polarization. The corresponding wave functions at $k=0$ are localized at the octagonal defect, but each one at a different local sublattice, like the TB wave functions in Fig. 3(c). However, for $k>\frac{2 \pi}{3}$ the two occupied flatbands below the Fermi energy have the same spin polarization. As a result, the calculated magnetization is almost three times weaker than in the previous case, being approximately $\sim 0.6 \mu_{B}$.

\section{B. Defect lines composed of alternating octagons and double pentagons}

Let us now consider a system that has been recently studied both experimentally ${ }^{2}$ and theoretically: ${ }^{21}$ a ZGNR with a defect line made of alternating octagons and pentagon pairs. This geometrical reconstruction of octagons into pentagons is driven by the $\sigma$ orbitals, but they do not need to be considered

(a)

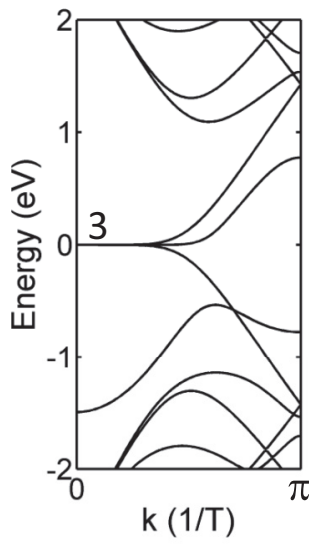

(c) (b)

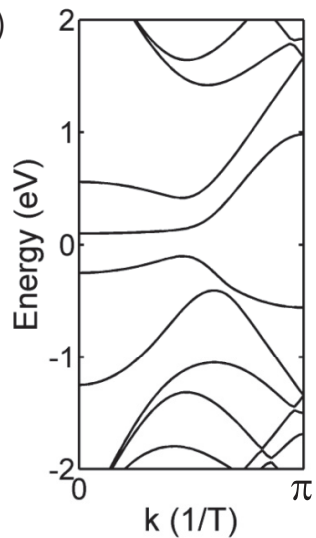

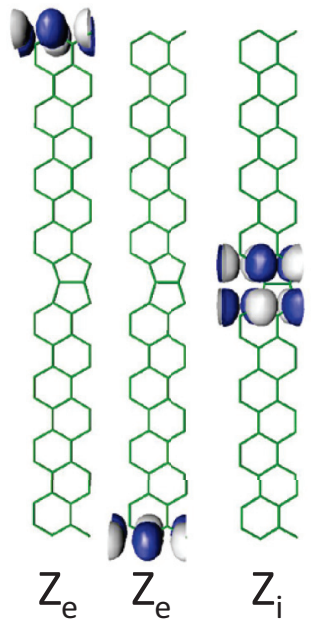

FIG. 4. (Color online) (a) Band structure of a ZGNR with a defect line composed of octagons and pentagon pairs, as depicted in Fig. 1(b), calculated within the TB model. (b) Band structure for the same system calculated with the Hubbard model. Spin-down and spin-up bands are degenerate in energy. (c) TB wave functions corresponding to the three zero-energy bands at $k=0$ found in the TB calculation. Blue (dark gray) and white colors represent positive and negative signs of the wave function, respectively.

here because their energies are much below the Fermi level. Due to this reconstruction, a new bond appears between pairs of the Klein-like nodes, which now have coordination number $3,2,11,34$ as shown in Fig. 1(b). Due to the presence of the pentagons there is a local sublattice mixing at the defect line. The band structure for the unrolled system within the TB approximation is shown in Fig. 4(a). The wave functions at $k=0$ corresponding to the flatbands are presented in Fig. 4(c).

As expected, the state that was localized at the Klein-like atoms in the previously studied system moves away from the Fermi energy due to the newly formed bond. However, the other state located at the zigzaglike rows of atoms does not change much. As its wave function is already zero at the newly connected Klein nodes, this state is not affected by the formation of the pentagons. Therefore, the corresponding band is much less affected by the on-site Coulomb interaction than the states localized at the GNR edges, which split up and down away from zero energy due to the electronic repulsion. This can be seen in the bands calculated within the Hubbard model, shown in Fig. 4(b). For $k=0$ the flattest band at Fermi energy 
is localized at the octagon. Our results close to the Fermi energy agree with those shown in Ref. 9 obtained with a density functional approach. The outer edges allow for localization of both the spin-down and the spin-up functions at the same nodes without large energy splitting between the corresponding bands, which is typical for zigzag nanoribbons. The flattest band at the Fermi energy, i.e., the one localized at octagons, is also a resonance between the two states belonging to the so-called inner zigzag "edges," as for the pure octagon cases, and therefore is not being affected much by the connection of the Klein-like modes forming the pentagons.

Let us finally consider the nanotube with the doublepentagon-octagon defect line. As done before, by rolling up the ribbon with the defect line, we obtain an $(8,8)$ armchair nanotube with a line of octagon and pentagon pair defects along the tube axis. This type of nanotube was previously studied by Okada and co-workers. ${ }^{35}$ When connecting the outer zigzag edges, the corresponding localized states disappear and only one flatband for $k<\frac{2 \pi}{3}$ survives in the vicinity of the Fermi energy, ${ }^{36}$ as depicted in Fig. 5(a). The TB wave function corresponding to this flat band is shown in Fig. 5(c), being localized at the octagon. The inclusion of the on-site Coulomb term in the Hubbard model [Fig. 5(b)] changes the spectrum negligibly, and all the bands remain spin degenerate. (a)

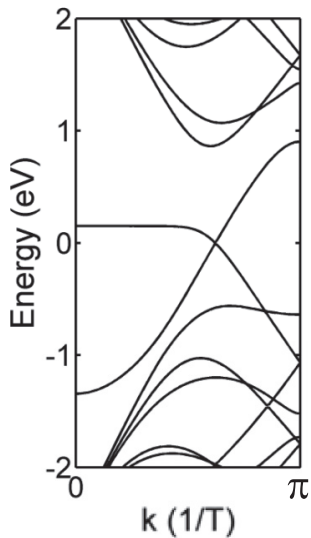

(b)

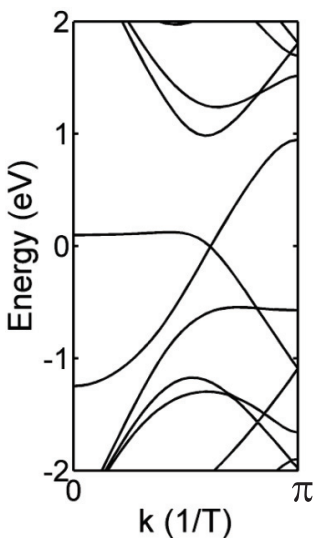

(c)

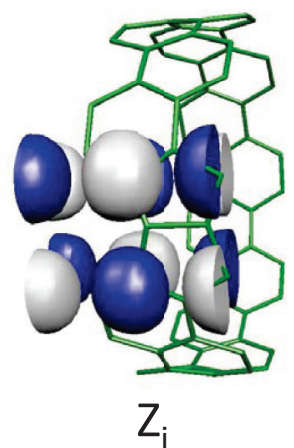

FIG. 5. (Color online) (a) Band structure of an $(8,8)$ armchair nanotube (rolled-up ZGNR) with a defect line composed of alternating octagons and pentagon pairs calculated with the TB approximation. (b) Band structure calculated within the Hubbard model. (c) TB wave function at $k=0$ of the flatband localized at the octagonal ring.

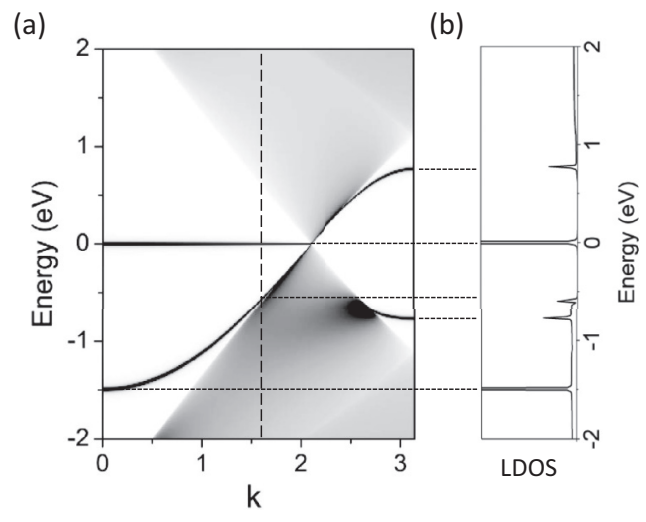

FIG. 6. (a) LDOS at the junction between two zigzag graphene half planes with an octagon-pentagon-pair defect line. Interface bands appear in the gap as dark lines, while the shaded area is the graphene band continuum. Vertical long dashed line marks $k=\pi / 2$. (b) LDOS at the junction between two $(8,0)$ nanotubes with an octagon-pentagon-pair junction. Horizontal dotted lines mark the correspondence between the energies of the interface bands of the graphene junction at the allowed wave vectors given by the Born-von Kármán boundary condition $k=0, \frac{\pi}{2}, \pi$ and the LDOS peaks for the $(8,0)$ nanotube junction. The LDOSs in (a) and (b) are calculated at the eight nodes of the octagon of the graphene and nanotube junctions, respectively.

\section{Junction between zigzag nanotubes made of alternating octagons and double pentagons}

In the previous section we first considered a ribbon made of two zigzag ribbons connected by an octagon-double-pentagon defect line. Next, we rolled up that system, getting an armchair carbon nanotube with a line of octagons and pentagon pairs along the tube axis. Now we want to roll up the initial ribbon in the perpendicular direction, in order to obtain two zigzag nanotubes joined by a sequence of octagons and pentagon pairs.

We start by letting the width of the initial ribbon tend to infinity. This yields an octagon-pentagon-pair junction between two graphene half planes, one with a zigzag edge and the other with a Klein-like edge. This system can be considered as a junction between two pure zigzag graphene semi-infinite half planes joined by a sequence of octagons and pentagon pairs [see Fig. 1(b)]. Figure 6(a) shows the calculated LDOS at such a junction. It is clear that it is a limiting case of Fig. 4(a) for infinite ribbon width.

By cutting a strip perpendicular to the defect line and rolling it up, a junction between two zigzag carbon nanotubes is obtained. Denoting the width of the strip by the number of zigzag nodes, a junction between two $(2 n, 0)$ zigzag nanoribbons can be made by rolling a strip of width $2 n$ (notice that since the unit cell of the junction contains one octagon and a pentagon pair, the smallest number of zigzag nodes in such a unit cell is 2). In principle, the interface states of these junctions are located by calculating the LDOS for each system. However, instead of performing a calculation for each $n$, we can easily find the number of interface states, their energies, and the relative strengths of localization at the junction by applying the Born-von Kármán boundary condition to the interface bands shown in Fig. 6(a), as in Ref. 16, where junctions 
between $(n, n)$ and $(2 n, 0)$ tubes were studied. Likewise, the energies of the nanotube interface states are determined by the crossings between the interface bands of the graphene junction in Fig. 6(a) and vertical lines marking wave vectors given by the Born-von Kármán boundary condition (Ref. 16)

$$
k=\frac{2 \pi m}{n}, \quad m=0, \ldots, n-1 .
$$

As an example, for a hypothetical junction between two $(2,0)$ nanotubes, there is only one allowed wave vector, $k=0$, yielding two gap states: one at $E=0$ and another at $E=-1.5 \mathrm{eV}$. The relative intensity of the LDOS for these energies gives an idea about the localization strength at the junction.

For comparison we have calculated the LDOS for the junction between two $(8,0)$ nanotubes $(n=4)$. It is shown in Fig. 6(b). The allowed wave vectors are therefore $k=$ $0, \frac{\pi}{2}, \pi, \frac{3 \pi}{2}$ (but only three in the irreducible BZ). The highest peak is at $E=0$, being much higher than the rest. The relative strengths of the peaks in the LDOS for the nanotube junction in Fig. 6(b) are the same as those in graphene shown in Fig. 6(a), with a correction for the peak at $E=0$, since it corresponds to two states, namely, those at $k=\frac{\pi}{2}$ and $\pi$. It is interesting to note that only two states, those with $E=0$, are localized exactly at the junction. The state at $E=-0.6 \mathrm{eV}$ is slightly delocalized, while the other three states are strongly delocalized. Additionally, there are two localized states with energies $E=-1.5 \mathrm{eV}$ and $E \approx \pm 0.77 \mathrm{eV}$ in the nanotube junction. They appear in the nanotube band continuum, but stem from the interface bands shown in Fig. 6(a).

\section{Explanation of the results presented in Secs. III A and III B}

In Ref. 17 a series of hybridization rules was presented, allowing for an explanation of the existence and degeneracy of the zero-energy flatbands localized in graphene ribbons with arbitrary edges. These rules are based on (a) band folding and (b) splitting of degenerate flatbands localized at different graphene sublattices whenever their wave functions overlap, i.e., when they are connected by a nearest-neighbor hopping. As in some of the systems studied here the lattice is no longer bipartite, the criterion will be just whether a nearest-neighbor hopping is set between atoms with a non-negligible wave function amplitude.

Let us apply those rules to explain all the results obtained in the previous sections III A and III B. We adopt a construction technique schematically shown in Fig. 7. We start from two zigzag GNRs, which result from horizontally cutting the ribbon, similar to the one shown in Fig. 1(a) but much wider, along the defect line. This yields two ZGNRs, one of them having Klein edge atoms at one side. The schematic band structures for such two ribbons are shown in Fig. 7(a). When these two ribbons are connected, no splitting of the zero-energy bands occurs. This is because all the states are either localized at opposite outer edges of the ZGNR (the two $Z_{e}$ bands), or appear in separate regions of the Brillouin zone ( $K$ and $\left.Z_{i}\right)$. This is visualized in Fig. 7(b).

Now we want to connect every second Klein-like atom to form pairs of pentagons. To this end, we first double the unit cell, so the Brillouin zone has to be folded, yielding the (a)
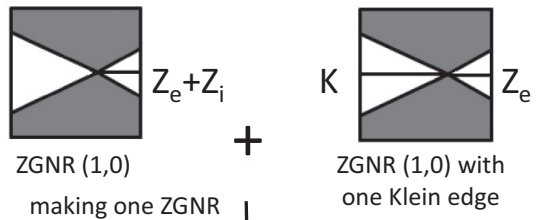

(b) with octagonal DL

(c)

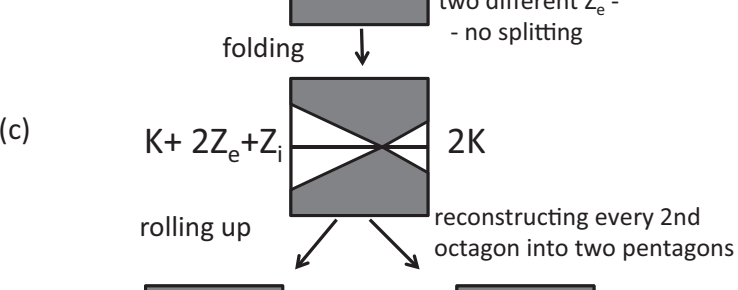

(dl)

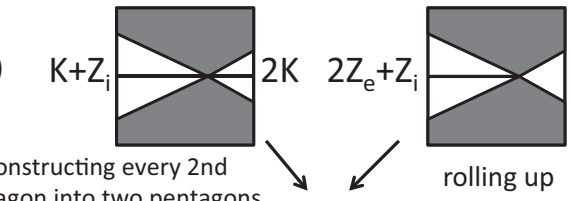

(e)

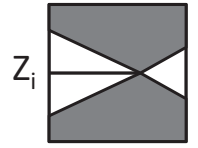

FIG. 7. Schematic band structures close to the Fermi energy for the systems investigated in Sec. III. Shaded regions stand for the band continua in case of very wide ribbons. $Z_{e}$ marks states localized at the outer zigzag edges, $Z_{i}$ marks states localized at the internal zigzag edges forming the octagon, and $K$ represents Klein edge-localized states. (a) Two independent ZGNRs, one of them with Klein edges at one side (right). (b) Two ZGNRs connected to form a ZGNR with an octagon-only line of defects. (c) ZGNR with an octagon defect line, but the band structure is folded from the previous case (b), because the real-space unit cell has twice the length. (dl) Rolled-up ZGNR with octagons only. (dr) ZGNR with octagon-double-pentagon defects (i.e., with Klein nodes connected). (e) Armchair nanotube with a line of defects composed of octagons and pentagon pairs.

band structure shown in Fig. 7(c). Connecting the Klein-like atoms to form the pentagons moves the $K$ bands away from the Fermi energy (see also Fig. 6) due to the newly formed bond, as shown in Fig. 7(dr). If we further roll up the ribbon into a CNT, the two $Z_{e}$ bands hybridize and disappear from the zero-energy region, as shown in Fig. 7(e). The same result can be obtained from stage (c), first rolling up the ribbon with octagons only, which yields Fig. 7(dl), and then forming the pentagons, ending up in Fig. 7(e). Note that the degeneracies of the zero-energy bands presented in Figs. 7(c), 7(dl), 7(dr), and 7 (e) without considering spin are the same as those indicated in panels (a) of Figs. 2-5, respectively.

\section{SUMMARY AND CONCLUSIONS}

We have investigated graphene nanoribbons and carbon nanotubes containing defect lines made of successive octagons or octagons and pentagon pairs. We have shown that the 
appearance of localized flatbands at the Fermi energy may be easily explained using the hybridization rules introduced in Ref. 17. One of the localized states is a resonance between two states belonging to the inner zigzag edges which conform to the line of defects. This state is robust and appears at the Fermi level in all the systems investigated. Therefore, octagonal defect lines indicate reactivity sites in defected graphene.

We extended our research to zigzag nanotubes connected by octagons and pentagon pairs. We have shown that all the nanotube interface states are related to the localized bands which appear in graphene with an octagon-pentagon-pair defect line. The number of these interface states and their energies can be easily deduced by applying the folding rule described in Ref. 16. Our approach gives a unified view of the origin and appearance of states localized at octagonal defect lines, relating them to graphene edge states.

Finally, we have included also the on-site electron-electron interaction effects by employing a Hubbard model, and we have investigated their influence on the localized zero-energy states. The spin-up and spin-down bands split only when there is no sublattice mixing at the nodes where they are localized. We have shown that such structures may present spontaneous magnetization.

\section{ACKNOWLEDGMENTS}

This work was supported by the Polish National Science Center (Grant No. DEC-2011/03/B/ST3/00091), the Basque Departamento de Educación and the UPV/EHU (Grant No. IT-366-07), the Spanish Ministerio de Innovación, Ciencia y Tecnología (Grant Nos. TEC2007-68065-C03-03 and FIS2010-19609-C02-02), and the ETORTEK research program (NANO-IKER Grant No. IE11-304) funded by the Basque Departamento de Industria and the Diputación Foral de Guipuzcoa. A.A. and L.C. acknowledge the Nicolaus Copernicus University in Torun for the generous hospitality during their visits.
${ }^{1}$ P. Y. Huang, C. S. Ruiz-Vargas, A. M. van der Zende, W. S. Whitney, M. P. Levendorf, J. W. Kevak, S. Garg, J. S. Alden, C. J. Hustedt, Y. Zhu et al., Nature (London) 469, 389 (2011).

${ }^{2}$ J. Lahiri, Y. Lin, P. Bozkurt, I. I. Oleynik, and M. Batzill, Nat. Nanotechnol. 5, 326 (2010).

${ }^{3}$ Q. Yu, L. A. Jauregui, W. Wu, R. Colby, J. Tian, Z. Su, H. Cao, Z. Liu, D. Pandey, D. Wei et al., Nat. Mater. 10, 443 (2011).

${ }^{4}$ S. Malola, H. Häkkinen, and P. Koskinen, Phys. Rev. B 81, 165447 (2010).

${ }^{5}$ O. V. Yazyev, R. B. Capaz, and S. G. Louie, Phys. Rev. B 84, 115406 (2011).

${ }^{6}$ Y. Liu and B. Jakobson, Nano Lett. 10, 2178 (2010).

${ }^{7}$ O. V. Yazyev and S. G. Louie, Nat. Mater. 9, 806 (2010).

${ }^{8}$ D. Gunlycke and C. T. White, Phys. Rev. Lett. 106, 136806 (2011).

${ }^{9}$ X. Lin and J. Ni, Phys. Rev. B 84, 075461 (2011).

${ }^{10}$ S. S. Alexaandre, A. D. Lúcio, A. H. C. Neto, and R. W. Nunes, Nano Lett. 12, 5097 (2012).

${ }^{11}$ L. Kou, C. Tang, W. Guo, and C. Chen, ACS Nano 5, 1012 (2011).

${ }^{12}$ X. Jia, M. Hofmann, V. Meunier, B. G. Sumpter, J. CamposDelgado, J. M. Romero-Herrera, H. Son, Y.-P. Hsieh, A. Reina, J. Kong et al., Science 323, 1701 (2009).

${ }^{13}$ X. Wang and H. Dai, Nat. Chem. 2, 661 (2010).

${ }^{14}$ D. V. Kosynkin, A. L. Higginbotham, A. Sinitskii, J. R. Lomeda, A. Dimiev, B. K. Price, and J. M. Tour, Nature (London) 458, 872 (2009).

${ }^{15}$ L. Jiao, L. Zhang, X. Wang, G. Diankov, and H. Dai, Nature (London) 458, 877 (2009).

${ }^{16}$ H. Santos, A. Ayuela, W. Jaskólski, M. Pelc, and L. Chico, Phys. Rev. B 80, 035436 (2009).

${ }^{17}$ W. Jaskólski, A. Ayuela, M. Pelc, H. Santos, and L. Chico, Phys. Rev. B 83, 235424 (2011).

${ }^{18}$ This condition is exact in the $\pi$-electron tight-binding approximation.

${ }^{19}$ J. Song, H. Liu, H. Jiang, Q.-F. Sun, and X. C. Xie, Phys. Rev. B 86, 085437 (2012).
${ }^{20}$ J. Liwei, Y. Guodong, G. Wenzhu, L. Zhe, and Z. Yisong, Phys. Rev. B 86, 165433 (2012).

${ }^{21}$ M. Kan, J. Zhou, Q. Sun, Q. Wang, Y. Kawazoe, and P. Jena, Phys. Rev. B 85, 155450 (2012).

${ }^{22}$ T. Hu, J. Zhou, J. Dong, and Y. Kawazoe, Phys. Rev. B 86, 125420 (2012).

${ }^{23}$ L. Chico, L. X. Benedict, S. G. Louie, and M. L. Cohen, Phys. Rev. B 54, 2600 (1996).

${ }^{24}$ We label CNTs with their unrolled circumference vector on the graphene plane, with the graphene lattice vectors forming an angle of $60^{\circ}$.

${ }^{25}$ W. Jaskólski and L. Chico, Phys. Rev. B 71, 155405 (2005).

${ }^{26}$ A. Ayuela, L. Chico, and W. Jaskólski, Phys. Rev. B 77, 085435 (2008).

${ }^{27}$ M. P. López Sancho, M. C. Muñoz, and L. Chico, Phys. Rev. B 63, 165419 (2001)

${ }^{28}$ J. N. B. Rodrigues, P. A. D. Goncalves, N. F. G. Rodrigues, R. M. Ribeiro, J. M. B. Lopes dos Santos, and N. M. R. Peres, Phys. Rev. B 84, 155435 (2011).

${ }^{29}$ J. Fernández-Rossier and J. J. Palacios, Phys. Rev. Lett. 99, 177204 (2007).

${ }^{30}$ L. Yang, Ch.-H. Park, Y.-W. Son, M. L. Cohen, and S. G. Louie, Phys. Rev. Lett. 99, 186801 (2007).

${ }^{31}$ H. Santos, L. Chico, and L. Brey, Phys. Rev. Lett. 103, 086801 (2009).

${ }^{32}$ We employ the standard notation which denotes a zigzag ribbon by the number of zigzag chains across its width.

${ }^{33}$ E. H. Lieb, Phys. Rev. Lett. 62, 1201 (1989).

${ }^{34}$ D. J. Appelhans, L. D. Carr, and M. T. Lusk, New J. Phys. 12, 125006 (2010)

${ }^{35}$ S. Okada, K. Nakada, K. Kuwabara, K. Daigoku, and T. Kawai, Phys. Rev. B 74, 121412 (2006).

${ }^{36}$ As the carbon nanotube has topological defects, electron-hole symmetry is broken, so the flatband can move from $0 \mathrm{eV}$. The Fermi energy is at the crossing point where the flatband slightly bends due to the small diameter of the tube. 